Check for updates

Cite this: RSC Adv., 2017, 7, 35957

Received 16th June 2017

Accepted 4th July 2017

DOI: 10.1039/c7ra01642e

rsc.li/rsc-advances

\section{Narrow band resonance in the UV light region of a plasmonic nanotextured surface used as a refractive index sensor $\dagger$}

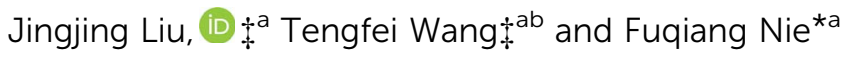

\section{Introduction}

Recently, the optical resonance of metallic nanostructures opens ups avenues for various applications such as chemical or biochemical sensing, ${ }^{1,2}$ surface-enhanced plasmonic sensing, ${ }^{3}$ metamaterials, ${ }^{4}$ optical waveguiding, ${ }^{5}$ and photonic applications. ${ }^{6}$ In particular, the metal-dielectric-metal (MDM) multilayer nanostructure by coupling exotic medium is of growing interest. ${ }^{7,8}$ MDM structures are a class of photonic metamolecules, ${ }^{9}$ which can support the propagation of the gap plasmon polariton (GPP) mode resulting from the coupling effect of surface polaritons, ${ }^{10,11}$ which can achieve the subwavelength confinement of light and have important applications in nanoscale optical circuits ${ }^{11}$ and enhanced optical intensity. ${ }^{12}$

The plasmonic resonance behavior in the visible-light region, ${ }^{7}$ near-infrared (NIR) region ${ }^{13}$ and even in the terahertz $(\mathrm{THz})$ region $^{14}$ have been demonstrated by various metal patterns to enhance the analyte sensitivity, which show that the resonance position is very sensitive on the dielectric surrounding medium. ${ }^{15}$ In general, researchers have obtained the effective refraction index of patterned perfect metal thin

\footnotetext{
${ }^{a}$ Division of Nanobionic Research, Suzhou Institute of Nano-Tech and Nano-Bionics, Chinese Academy of Sciences, Suzhou 215123, China. E-mail: fqnie2012@sinano.ac. cn; jjliu2015@sinano.ac.cn

${ }^{b}$ University of Chinese Academy of Sciences, Beijing 100049, China

$\dagger$ Electronic supplementary information (ESI) available. See DOI: 10.1039/c7ra01642e

$\ddagger$ These authors contributed equally.
}

films by means of either the Drude model or direct retrieval from the transmission and reflection coefficients. ${ }^{16}$ Herein, we report an aperiodic plasmic nanotextured surface with a metaldielectric-metal (MDM) multilayer nanostructure, which exhibits light-matter interactions overcoming the diffraction limit, enabling the refraction index application in the ultraviolet (UV) regime.

The electromagnetic plasmoic nanotextured surface (PNS) is associated with electric and magnetic fields propagating along a metal-dielectric interface and decaying exponentially in the horizontal and perpendicular directions. Most of the energy is then confined to the metal surface, which explains the remarkable sensitivity of the plasmoic nanotextured surface with respect to changes in the optical parameters at the metaldielectric boundary.

It is more challenging to detect volatile organic compounds (VOCs) with a small volume or in an unclosed system. Due to VOCs being non-ionic, non-fluorescent and easy to vapourize, the available analytical approaches used for detecting VOCs in microfluidic channels are quite essential. Davidsson et al. developed an ethanol sensor for microfluidic devices using chemiluminescence. ${ }^{17,18}$ It is a disadvantage that chemiluminescence is needed to be generated by an enzyme. In some other studies, VOCs are detected in an integrated optical Bragg grating refractometer. ${ }^{19}$ Although these methods do not require additional reagents, precise alignment of the light sources, waveguides, resonators, and detectors on the microfluidic devices are necessary. ${ }^{20}$ Thus, we have made use of plasmonic nanotextured nanomaterials and their surface sensitivities to 
demonstrate the refractive index sensing of various chemical solutions in an encapsulated polydimethylsiloxane (PDMS) microfluidic channel, named as plasmonic nanotextured devices (PNDs). The merits of PDMS-based microfluidic systems for biological assays are the reduction of reagent requirements, short analysis times, and portability. ${ }^{21,22}$ They can be manufactured to possess unique features including inlets, outlets, microvalves, microreactors, micromixers, and various microfluidic chambers. The fabricated microfluidic devices can be assembled in a short period of time without the requirement for expensive design software or clean-room facilities. Therefore, PDMS-based microfluidic systems provide a microenvironment and create a perfect sensing platform for trace chemical detection with several advantages including minimization, portability, minimal use of analytes/reagents and generation of waste, and faster reaction times. Using this technique, samples can be introduced in a continuous flow, allowing for the exposure of a light spot to a sufficient volume of sample.

To avoid the high absorption from water in NIR and middleinfrared (MIR) light regions, ${ }^{23}$ we explored the proposed PND structures to make the sensing in the near UV light region easily filled into the devices; moreover, in this study, we fabricated a novel plasmonic device working as a refractive index (RI) sensor for the 250-350 $\mathrm{nm}$ wavelength range, which is the first device for the ultra-violet (UV) light region.

\section{Materials and methods}

Fig. 1 shows the flow process used for the fabrication of the PNDs using natural lithography technology. First, $\mathrm{SiO}_{2}$ and gold (Au) thin-films with a thickness of 150/50 nm were deposited on a (100) n-type Si substrate using low-temperature chemical vapor deposition (LPCVD) and electron-beam evaporation, respectively, as shown in Fig. 1(a). Second, the sample was

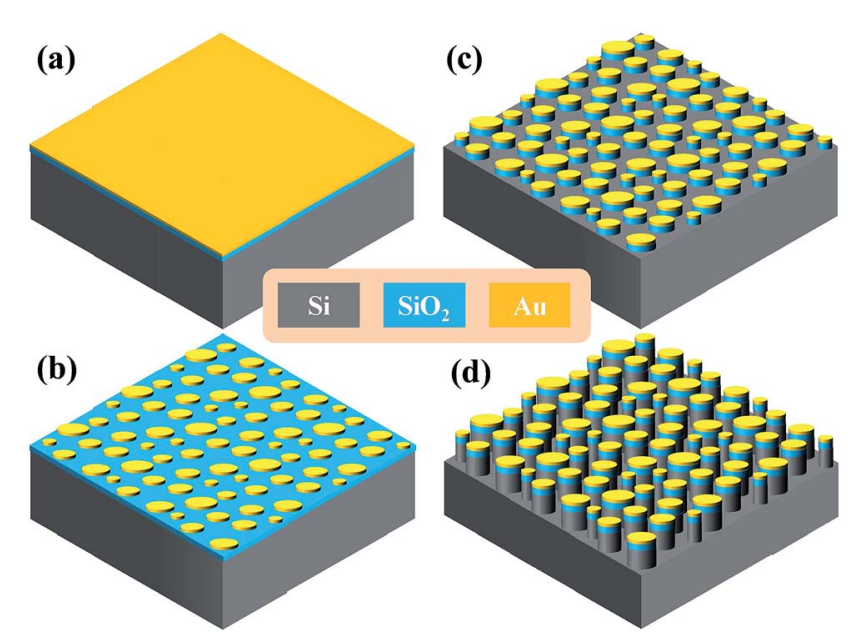

Fig. 1 The flow process used for the fabrication of the PNDs. (a) $\mathrm{SiO}_{2}$ and Au thin-films were deposited on a (100) n-type Si substrate. (b) The samples were annealed at $800^{\circ} \mathrm{C}$. (c) The samples were patterned by etching $\mathrm{SiO}_{2}$ to form the metal/dielectric structures. (d) The samples were patterned by etching $\mathrm{Si}$ to form the metal/dielectric/semiconductor structures. annealed at $800^{\circ} \mathrm{C}$ at $250 \mathrm{mTorr}$ in an $\mathrm{Ar}$ atmosphere for $20 \mathrm{~min}$ to form $\mathrm{Au}$ nanoparticles with a grain size in the range of 100$150 \mathrm{~nm}$ (Fig. 1(b)). Each island (i.e. grain) of the Au nanoparticles functioned as an individual hard mask for subsequent etching. The sample was vertically etched on the $\mathrm{SiO}_{2}$ thin-film surface using a mixed gas of $\mathrm{SF}_{6} / \mathrm{CHF}_{3}$ at $130 \mathrm{sccm}$ via reactive ion etching (RIE) at $5 \mathrm{mTorr}$ and a source power of $80 \mathrm{~W}$ for 1 min as shown in Fig. 1(c). Thus far, the devices were composed of metal/dielectric structures and denoted as PND-1. Sequentially, the samples were etched on the Si substrate with $\mathrm{SF}_{6}$ gas at $15 \mathrm{sccm}$ via RIE at 1000 mTorr for $120 \mathrm{~s}$ to form the metal/dielectric/semiconductor structures as shown in Fig. 1(d), denoted as PND-2.

To verify the VOS sensing characteristics using PND-1 and PND-2, the devices were encapsulated in a PDMS-based microfluidic channel and characterized using a UV-vis spectrometer (Ocean Optics Ltd. Co., Maya2000 Pro.). The fabrication process of the PDMS-based microfluidic system is illustrated as follows. PDMS prepolymer and curing agent (10:1 w/w, Sylgard 184, Dow-Corning) were mixed at a $10: 1$ ratio and degassed under vacuum for $30 \mathrm{~min}$. The mixture was poured over an SU-8 mold (thickness: $1 \mathrm{~mm}$, length: $10 \mathrm{~mm}$, width: $10 \mathrm{~mm}$ ) on a glass slide (length: $76.2 \mathrm{~mm}$; width: $25.4 \mathrm{~mm}$ ) master and then baked at $80{ }^{\circ} \mathrm{C}$ for $1 \mathrm{~h}$ to create the microchannel. After curing, a piece of PDMS microchannel was formed and peeled from the glass master. To complete the fabrication of the sensing system, the PDMS microchannel was drilled with holes, $5 \mathrm{~mm}$ in diameter for the inlet and outlet of the microfluidic system, and then plasma bonded to the Si substrate containing the pre-patterned plasmonic nanotextured surface. Finally, it was assembled with appropriate connectors, such as Tyron tubes, syringe, and syringe pump to form a microfluidic system. A schematic of the experimental set-up is shown in Fig. 2. The inlet and outlet of the PDMS fluidic channel were formed by having Tyron tubes inserted for sample delivery. The fluidic channel was connected to a syringe, via an inlet tube, and syringe pump, which was used to drive the various vOS alternately. The injected volume was kept at $100 \mu \mathrm{L}$ with a flow rate of $1 \mathrm{~mL} \mathrm{~min}^{-1}$ for all the VOS. These liquids give a good spread of refraction index values, enabling us to investigate the sensitivities of the plasmonic sensing with various VOS samples.

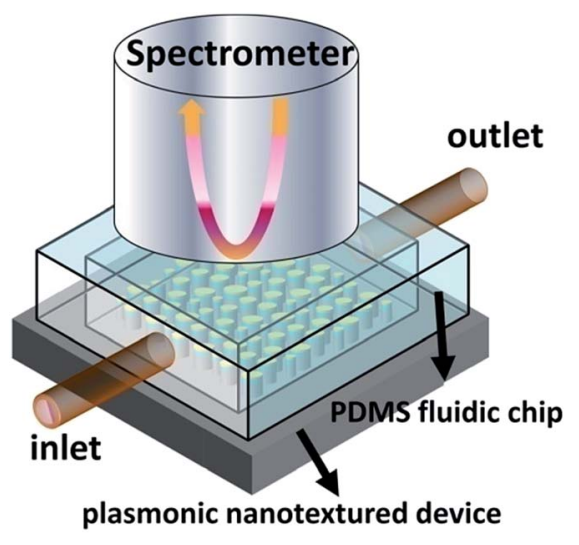

Fig. 2 A schematic of the microfluidic measurement set-up. 


\section{Results and discussion}

Fig. 3 shows the cross-sectional views of the PNDs using fieldemission scanning electron microscopy (SEM) system. It can be seen that the aperiodic nanostructures were distributed uniformly after the fabrication process for PND-1 and PND-2 as shown in Fig. 3(a) and (b), respectively. Fig. 3(c) and (d) show the cross-sectional SEM images in which the aperiodic nanostructures were measured to be in the period ranges of $\sim 300 \mathrm{~nm}$ and $\sim 500 \mathrm{~nm}$ in depth for PND-1 and PND-2, respectively. The multi-layers clearly show the nanostructures of PND-1 and PND2 composed of the metal/dielectric and metal/dielectric/ semiconductor structures. The height of the etched $\mathrm{SiO}_{2}$ in PND-1 is $150 \mathrm{~nm}$ as denoted by both L1 and L2 in Fig. 3(c) and (d), respectively.

In our nanostructures, the dielectric layer of $\mathrm{SiO}_{2}$ in the medium structure can highly enhance the local electromagnetic intensity around the plasmonic $\mathrm{Au}$ NPs. In the wavelength region from $250-400 \mathrm{~nm}$, two resonant peaks were found in PND-2 while a single resonant peak was found in PND-1, resulting from the two types of surface plasmons between the two nanotextured surfaces. The first type may be generated by the Au nanoparticles, which could be excited by light to oscillate at the interface. Conduction electrons in metal nanomaterials could be coherently excited by incident light to oscillate collectively at metal/dielectric interfaces. ${ }^{24}$ As can be seen from Fig. 4, the first resonant peak_1 in PND-2 is weaker than the resonant peak in PND-1 and a little blue-shift is observed. The lower intensity of reflective of peak_1 in PND-2 could be explained by the deeper etching condition with around $500 \mathrm{~nm}$ of the total height; moreover, the blue-shift is also known to be caused by the deeper structure. ${ }^{25}$ The $\mathrm{SiO}_{2} / \mathrm{Si}$, which is the dielectric/semiconductor layer, may give rise to the second type of surface plasmon, which is shown in Fig. 4 as peak_2 in PND-2.
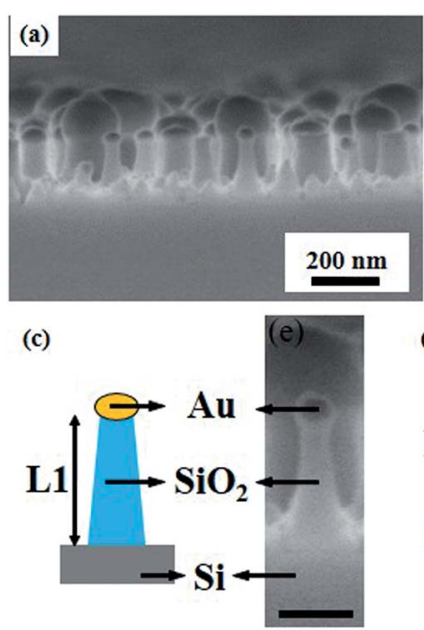

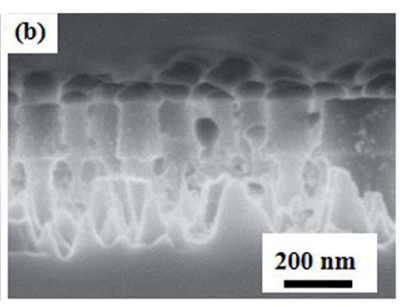

(d)

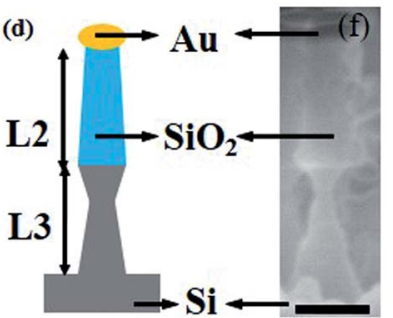

Fig. 3 (a) and (b) are the top SEM images and (c) and (d) are the crosssectional SEM images of PND-1 and PND-2, respectively. (e) and (f) are the corresponding unit nanostructural geometries of PND-1 and PND2 , respectively, where the scale bar is $100 \mathrm{~nm}$.
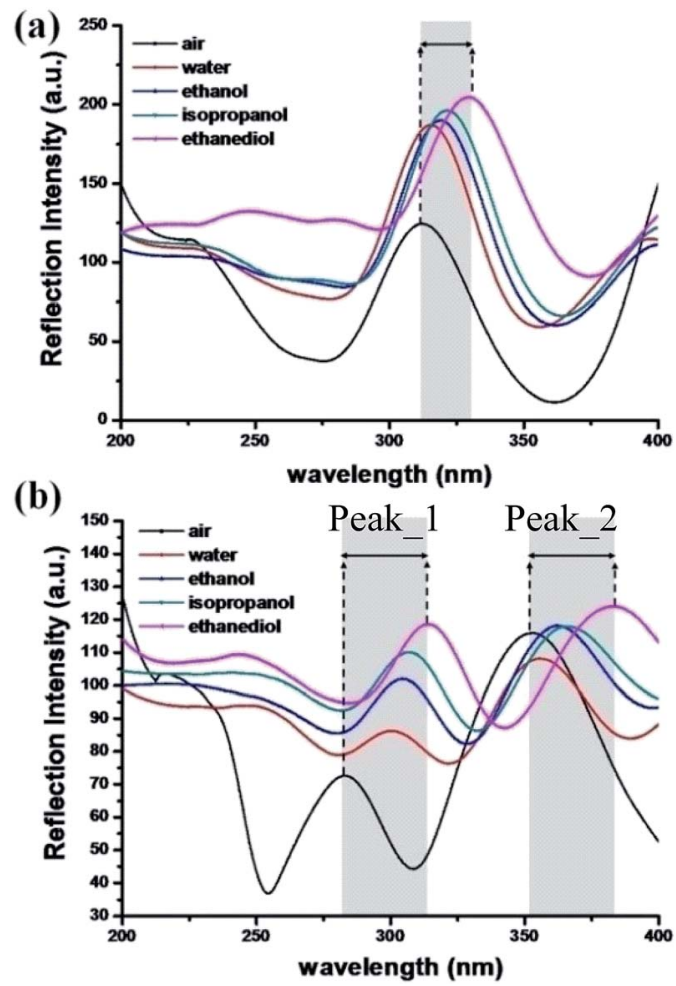

Fig. 4 The shift in the resonant wavelength of the PND-1 device (a) and PND-2 device (b) in various chemical solutions, respectively.

Fig. 4 shows the experimental results of the PND-1 and PND2 devices under various VOS. As can be seen, there is a significant red-shift at the resonances as the refraction index (RI) of the fluid filling the PDMS-based microchannel increases. In the absence of a chemical solution, i.e., $n=1$ (ambient air), the resonance is $312.5 \mathrm{~nm}$ for PND-1, while the two orders of PND-2 are located at $282.2 \mathrm{~nm}$ and $351.8 \mathrm{~nm}$. When the microfluidic channel was injected with DI water, ethanol, isopropanol, and ethanediol, the resonances were shifted from $312.2 \mathrm{~nm}$ (air) to $315.2 \mathrm{~nm}$ (water), $312.2 \mathrm{~nm}$ (air) to $320.1 \mathrm{~nm}$ (ethanol), $312.2 \mathrm{~nm}$ (air) to $321.2 \mathrm{~nm}$ (IPA) and $312.5 \mathrm{~nm}$ (air) to $329.9 \mathrm{~nm}$ (ethanediol) for PND-1. Fig. 5 gives the resonance intensity variations among the different media or solution for peak_1 and peak_2 of PND-2. It is interesting that the variation of the resonances for the two peaks tend to approach the RI value increasing with the different chemical solutions. To further investigate the VOS sensing capabilities of PND-1 and PND-2, a $\mathrm{C}_{2} \mathrm{H}_{4}(\mathrm{OH})_{2}$ solution at different concentrations was chosen and injected into the PDMS-based microfluidic channel (Fig. S1, ESI†).

To clarify the sensitive capability of PNDs, the resonant shifting of the various chemical solutions was plotted against their respective refractive indices in Fig. 6. Notably, the RI values vary as the wavelength changes; thus, after a slit resonant shift, the RI value will be altered sequentially. We added the range of the RI change, which we referred to from the website of the refractive index database. ${ }^{26}$ Apparently, PND-2 shows a larger resonant shift than PND-1. Furthermore, the maximum 


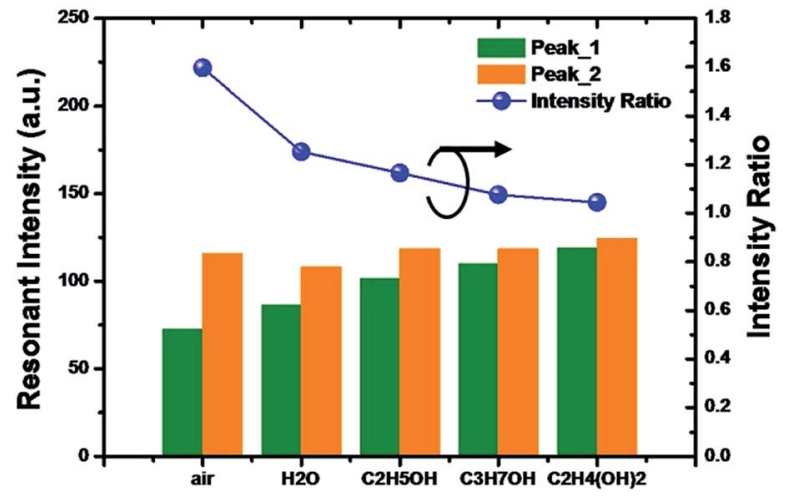

Fig. 5 The variation in the resonant intensity among different media or solution for peak_1 and peak_2 of PND-2.

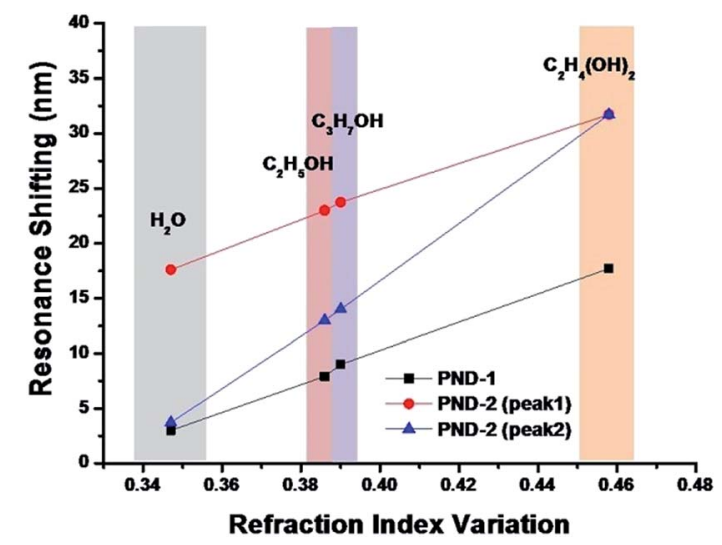

Fig. 6 The relationship between the variation in refractive index and shift in the resonance of PND-1 and PND-2 (the refraction index value refers to database ${ }^{26}$ ).

resonant shift was $31.7 \mathrm{~nm}$, which is larger than the reported resonant RI shift of $15 \mathrm{~nm}^{27}$

The sensitivity $\left(\mathrm{nm} \mathrm{RIU}{ }^{-1}\right.$ ) of the plasmonic nanotextured device is the shift in the resonant wavelength per unit change of the refraction index. Table 1 summarizes the sensing performance of the PNDs used as a refractive index sensor. Comparing the sensitivity ( $\mathrm{nm} \mathrm{RIU}{ }^{-1}$ ) of peak_1 and peak_2 of PND-2 and PND-1, they are from $50.4 \mathrm{~nm} \mathrm{RIU}^{-1}$ to $68.6 \mathrm{~nm} \mathrm{RIU}^{-1}$ for peak_1 of PND-2, from $10.8 \mathrm{~nm} \mathrm{RIU}^{-1}$ to $70.8 \mathrm{~nm} \mathrm{RIU}^{-1}$ for peak $\_2$ of PND-2, and $8.6 \mathrm{~nm} \mathrm{RIU}^{-1}$ to $38.6 \mathrm{~nm} \mathrm{RIU}^{-1}$, respectively. This shows that peak_1 of PND-2 improved the sensitivity around 1.6fold and 2.5-fold on average better than that of peak_2 of PND-2 and PND-1, respectively. For sensing different concentrations of $\mathrm{C}_{2} \mathrm{H}_{4}(\mathrm{OH})_{2}$, the performance of the sensitivities are summarized in Table S1 (ESI $\dagger$ ). A significant narrow FWHM (Full-Width at Half-Maximum) was found in our study: $\sim 28 \mathrm{~nm}$ for PND-1, $\sim 20 \mathrm{~nm}$ for peak $\_1$ of PND-2 and $\sim 30 \mathrm{~nm}$ for peak $\_2$ of PND-2. This indicates that low energy losses excited from plasmon resonant are contributed by the aperiodic structure. ${ }^{28}$ Moreover, the coupling deeper structures of PND-2 with metal/dielectric/ semiconductor show the sharp FHMW $\sim 20 \mathrm{~nm}$ for peak_1. PND-2 shows a high sensitivity that might be attributed MDM, resulting from the coupling effect of surface waves. It will further
Table 1 The sensing performance of the PNDs used as a refractive index sensor

\begin{tabular}{ccccc}
\hline Solution & $\begin{array}{l}\text { Resonant } \\
\text { shift }(\Delta)\end{array}$ & FWHM & $\begin{array}{l}\text { Sensitivity } \\
\left(\text { nm RIU }^{-1}\right)\end{array}$ & FOM \\
\hline PND-1 & & & & \\
$\mathrm{H}_{2} \mathrm{O}$ & 3 & 29 & 8.6 & 0.3 \\
$\mathrm{C}_{2} \mathrm{H}_{5} \mathrm{OH}$ & 7.9 & 28 & 20.5 & 0.7 \\
$\mathrm{C}_{3} \mathrm{H}_{7} \mathrm{OH}$ & 9 & 28 & 22.5 & 0.8 \\
$\mathrm{C}_{2} \mathrm{H}_{4}(\mathrm{OH})_{2}$ & 17.7 & 29 & 38.6 & 1.3 \\
& & & & \\
PND-2 (peak_1) & & & & \\
$\mathrm{H}_{2} \mathrm{O}$ & 17.6 & 19 & 50.4 & 2.7 \\
$\mathrm{C}_{2} \mathrm{H}_{5} \mathrm{OH}$ & 21.2 & 19 & 54.5 & 2.9 \\
$\mathrm{C}_{3} \mathrm{H}_{7} \mathrm{OH}$ & 23.7 & 21 & 58.2 & 2.8 \\
$\mathrm{C}_{2} \mathrm{H}_{4}(\mathrm{OH})_{2}$ & 31.7 & 22 & 68.6 & 3.1 \\
& & & & \\
PND-2 $($ peak_2 $)$ & & & & \\
$\mathrm{H}_{2} \mathrm{O}$ & 3.7 & 32 & 10.8 & 0.3 \\
$\mathrm{C}_{2} \mathrm{H}_{5} \mathrm{OH}$ & 10.3 & 31 & 27.2 & 0.9 \\
$\mathrm{C}_{3} \mathrm{H}_{7} \mathrm{OH}$ & 14 & 31 & 35.4 & 1.1 \\
$\mathrm{C}_{2} \mathrm{H}_{4}(\mathrm{OH})_{2}$ & 31.7 & 37 & 70.8 & 1.9 \\
\hline
\end{tabular}

prove that the hybridized resonance can enhance the field confinement of the reflected light. ${ }^{25}$ According to the standingwave resonance mode, energy confinement in the nanocavity can arouse a significant resonance narrowing. ${ }^{25}$

In order to provide better quantification, the figure-of-merit (FOM) is another important factor used to determine the capability of a chemical sensor. It is defined as the sensitivity value divided by the resonance line width at half-maximum, i.e. FOM $=(\Delta \lambda / \Delta n) /$ FWHM, where $\Delta \lambda / \Delta n$ is the change in the resonant wavelength per refraction, index unit and the FWHM is the full width at half maximum of the resonance. ${ }^{29}$ This FOM was calculated for each sample to evaluate the performance of the devices for refraction index sensing. Correspondingly, we can obtain the highest value of FOM as 3.1, for peak_1 of PND-2 sensing ethanediol, which is around 2.3-fold higher than PND1, and around 1.6-fold higher than peak_ 2 of PND-2 sensing the same chemical solution, respectively. Finally, we demonstrated that our PND devices may serve as highly efficient plasmonic sensors in the $250-400 \mathrm{~nm}$ wavelength range.

In conclusion, we have demonstrated an effective chemical sensing approach using a plasmoic nanotextured surface integrated with a PDMS fluidic channel platform. The PND design has two major advantages. First, it can be easily fabricated using natural lithography technology. Second, the sensing medium is easily filled into the voids for plasmonic resonance sensor applications. Therefore, it facilitates the detection of refraction index changes. The experimental results indicate the feasibility of refraction index detection for different chemical solutions. This indicates a creation of a remote optical sensing mechanism using the chemical solutions with different refraction indexes to adjust the resonant wavelength of the PNDs. An ultranarrow FHMW of $\sim 20 \mathrm{~nm}$ was found in peak_1 of PND-2, indicating that the coupling deeper structures of with metal/ dielectric/semiconductor can improve the sensitivity. We believe that our MDM nanostructures can be exploited for further applications in enhanced spectroscopy. 


\section{Acknowledgements}

This study is financial supported by the Jiangsu Natural Science Youth Foundation (No. BK20160398) and Suzhou Science and Technology Project (No. SYG201633).

\section{References}

1 Y. Tian, et al., An Individual Nanocube-Based Plasmonic Biosensor for Real-Time Monitoring the Structural Switch of the Telomeric G-Quadruplex, Small, 2016, 12(21), 2913-2920.

2 M. T. Do, et al., Fabrication and Characterization of LargeArea Unpatterned and Patterned Plasmonic Gold Nanostructures, J. Electron. Mater., 2016, 45(5), 2347-2353.

3 M. Abb, et al., Surface-enhanced infrared spectroscopy using metal oxide plasmonic antenna arrays, Nano Lett., 2014, 14(1), 346-352.

4 S. A. Gregory, et al., Extreme Subwavelength Metal Oxide Direct and Complementary Metamaterials, ACS Photonics, 2015, 2(5), 606-614.

5 M. Fevrier, et al., Giant coupling effect between metal nanoparticle chain and optical waveguide, Nano Lett., 2012, 12(2), 1032-1037.

6 C. Lin, et al., Oxyfluoride Glass-Ceramics for Transition Metal Ion Based Photonics: Broadband Near-IR Luminescence of Nickel Ion Dopant and Nanocrystallization Mechanism, J. Phys. Chem. C, 2016, 120(8), 4556-4563.

$7 \mathrm{~J}$. Fan, et al., Standing-wave resonances in plasmonic nanoumberllar cavities for color generation and colorimetric refractive index sensor, Appl. Surf. Sci., 2016, 384, 534-538.

$8 \mathrm{~J}$. Chen, et al., Coupling between gap plasmon polariton and magnetic polariton in a metallic-dielectric multilayer structure, Phys. Rev. E: Stat., Nonlinear, Soft Matter Phys., 2011, 84, 026603.

9 C. Tserkezis, et al., Understanding artificial optical magnetism of periodic metal-dielectric-metal layered structures, Phys. Rev. B: Condens. Matter Mater. Phys., 2008, 78(16), 165114.

$10 \mathrm{~K}$. Park, et al., Study of the surface and bulk polaritons with a negative index metamaterial, J. Opt. Soc. Am. B, 2005, 22(5), 1016-1022.

11 K. Tanaka and M. Tanaka, Simulations of nanometric optical circuits based on surface plasmon polariton gap waveguide, Appl. Phys. Lett., 2003, 82(8), 1158.

$12 \mathrm{~J}$. Chen, et al., Optical bistability enhanced by highly localized bulk plasmon polariton modes in subwavelength metal-nonlinear dielectric multilayer structure, Appl. Phys. Lett., 2009, 94(8), 081117.

13 M. D. keshav, et al., Subpicosecond optical switching with a negative index metamaterial, Nano Lett., 2009, 9(10), 3565-3569.
14 D. K. Polyushkin, et al., THz generation from plasmonic nanoparticle arrays, Nano Lett., 2011, 11(11), 4718-4724.

$15 \mathrm{~N}$. Liu, et al., Planar metamaterial analogue of electromagnetically induced transparency for plasmonic sensing, Nano Lett., 2010, 10(4), 1103-1107.

$16 \mathrm{M}$. Ren, et al., Isotropic spiral plasmonic metamaterial for sensing large refractive index change, Opt. Lett., 2013, 38(16), 3133-3136.

17 R. Davidsson, et al., Microfluidic biosensing systems. Part I. Development and optimisation of enzymatic chemiluminescent micro-biosensors based on silicon microchips, Lab Chip, 2004, 4(5), 481-487.

18 C. F. Carlborg, et al., A packaged optical slot-waveguide ring resonator sensor array for multiplex label-free assays in labson-chips, Lab Chip, 2010, 10(3), 281-290.

19 D. J. Wales, et al., An integrated optical bragg grating refractometer for volatile organic compound detection, Sens. Actuators, B, 2016, 232, 595-604.

20 P. S. Nunes, et al., Refractive index sensor based on a $1 \mathrm{D}$ photonic crystal in a microfluidic channel, Sensors, 2010, 10(3), 2348-2358.

21 H. Somaweera, A. Ibraguimov and D. Pappas, A review of chemical gradient systems for cell analysis, Anal. Chim. Acta, 2016, 907, 7-17.

22 S. G. M. Uzel, et al., Simultaneous or Sequential Orthogonal Gradient Formation in a 3D Cell Culture Microfluidic Platform, Small, 2016, 12(5), 612-622.

$23 \mathrm{~J} . \mathrm{Xu}, \mathrm{K}$. W. Plaxco and S. J. Allen, Absorption spectra of liquid water and aqueous buffers between 0.3 and 3.72 THz, J. Chem. Phys., 2006, 124(3), 036101.

24 S.-Y. Ding, et al., Nanostructure-based plasmon-enhanced Raman spectroscopy for surface analysis of materials, Nat. Rev. Mater., 2016, 1(6), 16021.

$25 \mathrm{~J}$. Fan, et al., Standing-wave resonances in plasmonic nanoumbrella cavities for color generation and colorimetric refractive index sensor, Appl. Surf. Sci., 2016, 384, 534-538.

26 http:/www.refractiveindex.info/? shelf $=$ organic\&book $=$ ethanol\&page $=$ Sani.

27 S. G. Uzel, et al., Simultaneous or Sequential Orthogonal Gradient Formation in a 3D Cell Culture Microfluidic Platform, Small, 2016, 12(5), 612-622.

28 Y. Li, et al., Phase Separation of Silicon-Containing Polymer/ Polystyrene Blends in Spin-Coated Films, Langmuir, 2016, 32(15), 3670-3678.

29 L. J. Sherry, et al., Localized surface plasmon resonance spectroscopy of single silver nanocubes, Nano Lett., 2005, 5(10), 2034-2038. 